\title{
VARIACIÓN ESTACIONAL DE LA COMUNIDAD DE ESCARABAJOS Scarabaeinae (Coleoptera: Scarabaeidae) DE UN BOSQUE INUNDABLE AMAZÓNICO DE PERÚ
}

\section{SEASONAL VARIATION IN THE DUNG BEETLES COMMUNITY (Coleoptera: Scarabaeidae) FROM THE PERUVIAN AMAZON FLOODPLAIN}

\author{
Lidia Sulca Garro ${ }^{1}$ y Ana A. Huamantinco Araujo ${ }^{2}$
}

\begin{abstract}
Resumen
Los escarabajos Scarabaeinae cumplen un rol fundamental en la dinámica de los ecosistemas tropicales. Por ello, es importante conocer sobre su variación estacional en un bosque amazónico temporalmente inundable. Nuestro objetivo principal es conocer la variación de la riqueza, abundancia y composición de las comunidades de los escarabajos Scarabaeinae (Coleoptera: Scarabaeidae) en dos temporadas (lluvias y seca), comparandolos con dos tipos de cebos. Se efectuaron muestreos en ambas temporadas (seca e lluvias) empleando trampas de caída con dos tipos de cebos: fecas humanas y carroña de pescado. Se registraron 16422 individuos pertenecientes a 39 especies: 14431 pertenecientes a 35 especies en la temporada húmeda y 1991 pertenecientes a 28 especies en la temporada seca. Las especies dominantes fueron Ontophagus haematopus, Canthon quinquemaculatus y C. aequinoctialis. Se registraron diferencias significativas en abundancia entre ambas temporadas y los dos tipos de cebo, usando el modelo lineal generalizado (GLM). Por lo contrario, en la riqueza no muestran diferencias significativas. El análisis de similitud (ANOSIM), usando el método de escalamiento multidimensional (MDS) mostró diferencias significativas en la composición de escarabajos Scarabaeinae entre las dos temporadas, así como entre los dos tipos de cebo. La curva de acumulación de especies mostró una estimación del $94 \%$ de las especies esperadas. Se encontró una fluctuación marcada en la abundancia de los escarabajos Scarabaeinae debido a la temporalidad.

Palabras clave: riqueza, abundancia, temporada de lluvias, temporada seca.
\end{abstract}

\begin{abstract}
Scarabaeinae dung beetles play a fundamental role in the tropical ecosystem dynamics. Thus, it is important to study their seasonal variations in the amazon floodplain. Our main goal was to identify variations in richness, abundance and community structure in Scarabaeinae (Coleoptera: Scarabaeidae) dung beetles in two seasons (wet and dry), comparing two kinds of baits. Collections were made in both seasons (dry and rain) using human feces and putrid fish as bait traps. A total of 16422 specimens belonging to 39 species: 14431 belonging to 35 species in the rainy season and 1991 specimens belonging to 28 species in dry season were found. Dominant species were Ontophagus haematopus Harold 1875, Canthon quinquemaculatus Castelnau 1840, and Canthon aequinoctialis Harold 1868. Abundance compared between the two seasons and two kinds of baits, using the generalized linear model (GML), showed statistically significant differences. Otherwise, richness did not show significant difference. Analysis of similarity (ANOSIM), using the multidimensional scaling method (MDS), showed a significant difference of the composition of dung beetles community in the two seasons, as well as between the two kinds of baits. The species accumulation curve showed optimal sampling efforts, with more of $94 \%$ of expected species. We found a pronounced fluctuation of the dung beetles due to the temporality.
\end{abstract}

Key words: richness, abundance, wet season, dry season. 


\section{Introducción.}

Los escarabajos Scarabaeinae (Coleoptera Scarabaeidae) tienen un rol ecológico importante en varios procesos biológicos de los ecosistemas terrestres, como polinización, dispersión de semillas e incorporación de nutrientes al suelo (Estrada \& Coates-Estrada, 1991). También contribuyen en el ciclo de nutrientes al retornar al suelo los nutrientes almacenados en las fecas y cadáveres de los vertebrados (Steinfeld et al., 2006). Además, son capaces de remover huevos de helmintos de la superficie del suelo (Miller, 1954).

Para estos escarabajos el suelo es un factor clave para su ciclo de vida, pues allí es donde localizan su alimento, se desarrollan sus estadíos inmaduros y donde los nidos de varias especies son construidos (Halffter, 1991). Aunque es lógico pensar que la inundación del bosque en ciertos periodos del año los afecta significativamente. Sin embargo, se registró un aumento en la abundancia de algunas especies como Ontophagus haematopus Harold 1875, Canthon quinquemaculatus Castelnau 1840, Canthon aequinoctialis Harold 1868.

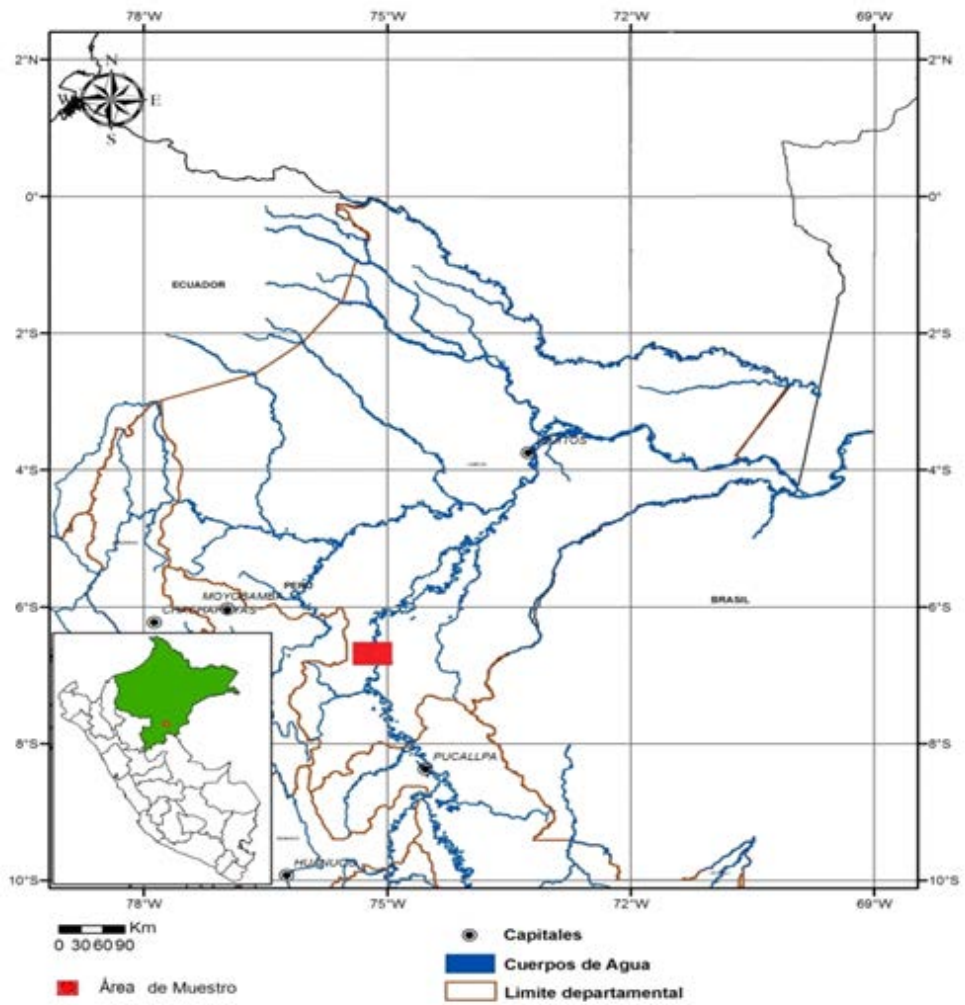

Figura 1. Área de localización del estudio. En verde, ubicación del departamento de Loreto; en cuadro rojo, ubicación del área de estudio ubicado en el Poblado Miguel Grau, provincia de Sarayacu, departamento de Loreto.
Las inundaciones de los Bosque de Llanura Meándrica del Perú generan considerable estrés a las plantas y animales, lo que genera adaptaciones para su supervivencia durante los largos periodos de total o parcial sumersión (Adis, 1988). Los artrópodos que habitan estos tipos de bosques, en particular, han desarrollado varias soluciones evolutivas a los periodos de pérdida de sus hábitats terrestres, como (1) quedándose cerca del límite del agua y moviéndose cuando advierten el ascenso del agua, (2) moviéndose al sotobosque no inundado o al dosel, (3) volando a biotopos secos adyacentes, y (4) desarrollando adaptaciones para resistir las inundaciones (Adis, 1986, 1988).

El objetivo de este trabajo es conocer la variación de la riqueza, abundancia y composición de las comunidades de los escarabajos Scarabaeinae (Coleoptera: Scarabaeidae) en dos temporadas (lluvias y seca), comparando dos tipos de cebos, en un bosque temporalmente inundable amazónico.

\section{Materiales y métodos.}

\section{Área de estudio}

Para conocer la estructura de la comunidad se hicieron las colectas a orillas del río Ucayali (6³7'27.40" S / 75³'54.10" O, 140 msnm), frente al Centro Poblado Miguel Grau, distrito de Sarayacu, provincia de Ucayali, Loreto (Figura 1) en un área aproximada de $8 \mathrm{~h}$. La zona se extiende dentro de la formación ecológica Bosque Húmedo Tropical (sensu ONE, 1976) y en un tipo de vegetación de Bosque de Llanuras Meándricas (sensu INRN, 1996). Estos bosques experimentan la influencia de los flujos estacionales del volumen de agua y la carga sedimentaria de las crecientes de los ríos, por lo que temporalmente se inundan generando cambios en la extensión del terreno, lo cual determina la presencia de especies vegetales particulares adaptadas a tales condiciones (IIAP, 2004), como catahuas, Hura crepitans, el machimangos, Eschweilera sp. lupunas, Chorisia integrifolia, capironas, Calycophyllum spruceanum, y varias especies de shimbillos (Inga spp.), así como lagartos caspi, Calophyllum brasiliense, moenas, Aniba sp. y cumalas, Virola sp.; asociadas a abundantes y exuberantes de palmeras pequeñas y medianas, lianas y epífitas, como los aguajes, Mauritia flexuosa, wasais, Euterpe precatoria, cashaponas, Socratea exorrhiza, huacraponas, Iriartea deltoidea, yarinas, Phytelephas macrocarpa, Wettinia sp., Geonoma sp. y Euterpe sp.

\section{Muestreo y procesamiento de muestras}

Las colectas fueron realizadas en dos temporadas: en enero (temporada de lluvias) y octubre (temporada seca) de 2013. Se establecieron dos líneas paralelas de 
trampas con una longitud aproximada de 300 m y separadas 50 m entre sí, cada una con diez trampas de caída (pit-fall) distanciadas $30 \mathrm{~m}$ una de otra (Silva, 2011; Villarreal et al., 2006). Se utilizaron dos tipos de cebos: fecas humanas (coprotrampas) y carroña de pescado (necrotrampas), se colocó en cada línea de trampa un tipo de cebo. Las trampas fueron revisadas en intervalos de $48 \mathrm{~h}$, momento en el cual eran retirados y colocados en bolsas de polipropileno y conservados en alcohol de 96\%; así mismo, se realizó el recebado de las trampas (Villarreal et al., 2006) en tres ocasiones más. No hubo pérdidas de trampas. Se consideró como unidad de muestreo al conjunto de ejemplares colectados en cada línea de trampas por 48 $h$, por lo que en total se tuvieron cuatro repeticiones para cada temporada y tipo de cebo. El considerar a cada línea de trampas como unidad de muestro es recomendado por diversos autores para estudios ecológicos con escarabajos Scarabaeinae (Quintero \& Roslin, 2005; Arellano et al. 2008).

La trampa pit-fall consiste en un recipiente de plástico enterrado al nivel del suelo, de un litro de capacidad, en cuyo interior se depositan aproximadamente $400 \mathrm{~cm}^{3}$ de agua más detergente líquido. Sobre la trampa, a unos $5 \mathrm{~cm}$ de altura, permanece suspendido el cebo (fecas humanas y carroña de pescado envuelto en una gasa cuadrada). En gabinete, los escarabajos fueron separados y preparados en alfileres entomológicos para proceder a la identificación. Para la identificación de los ejemplares se empleó la clave de Vaz-de-Mello (2011) y se consultó la colección entomológica del Museo de Historia Natural de la Universidad Nacional Mayor de San Marcos, donde fueron depositados los especímenes identificados.

En enero se registró $250 \mathrm{~mm}$ de precipitación, 85.9\% de humedad relativa promedio, una

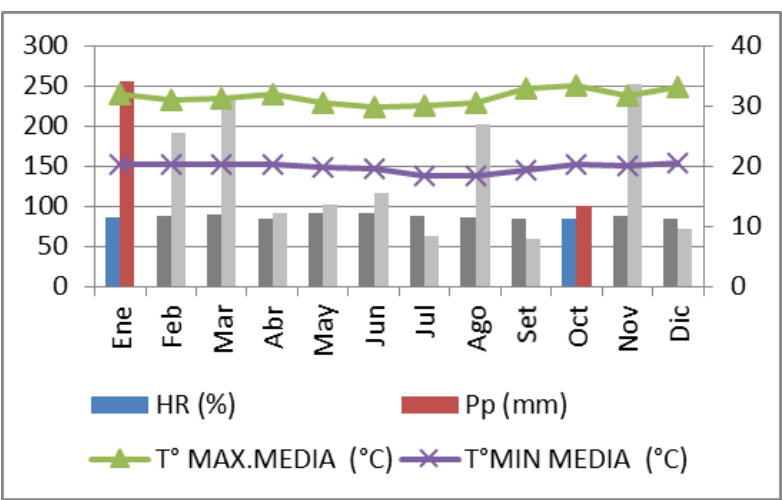

Figura 2. Datos climatológicos mensuales (HR: humedad, Pp: precipitación y $\mathrm{T}^{\mathrm{o}} \mathrm{MAX}$. MEDIA: temperatura máxima media, T ${ }^{\circ}$ MIN.MEDIA: temperatura mínima media) en La Estación Contamana, localizada a $80 \mathrm{~km}$ del área de estudio. SENAMHI (2013). Los datos de HR (azul) y Pp (rojo) fueron destacados en los meses de colecta. temperatura máxima de $32.0^{\circ} \mathrm{C}$ y una mínima de $20.2^{\circ}$, en tanto que en octubre se registró $100.2 \mathrm{~mm}, 84.1 \%$, $33.3^{\circ} \mathrm{C}$ y $20.2^{\circ} \mathrm{C}$, respectivamente (SENAMHI, 2013) (Figura 2).

Análisis de datos

Para determinar si existían diferencias significativas de la riqueza y abundancia se utilizaron regresiones en forma de modelos lineales generalizados (GLM), tomando en cuenta los factores de temporada y tipo de cebo, utilizando el paquete Statistica 8 (StatSoft Inc, 2007).

Con el fin de determinar la existencia de diferencias significativas en la composición de especies de escarabajos entre ambas temporadas (húmeda y seca) y entre los tipos de cebo (fecas humanas y vísceras de pescado podrido) se realizó el análisis de similitud (ANOSIM) de dos vías cruzado (con 999 permutaciones). Este análisis considera dos pruebas: en la primera, se prueba la hipótesis nula de que no hay diferencias entre temporadas; y en la segunda se prueba la hipótesis nula de que no hay diferencias entre tipos de cebo. Para cada prueba se calcula de forma independiente el estadístico Global $\mathrm{R}$, el cual varía entre 0 y 1 , e indica la relación que tiene la composición de especies con el factor. Los valores cercanos a 1 indican que la composición de especies está altamente relacionada con el factor, y los valores cercanos a cero indican que no hay relación. Además, se origina un nivel de significancia para rechazar o no la hipótesis nula considerando al 5\% como el nivel crítico (Clarke \& Warwick, 2001). Previamente al ANOSIM se construyó una matriz de similitud basada en el índice de Bray-Curtis con las abundancias relativas previamente transformadas como $\log (X+1)$. Para analizar gráficamente los patrones de distribución temporal y por tipo de cebo se aplicó el Método de Escalamiento Multidimensional (MDS)

Para la elaboración de las curvas de acumulación de especies, se usaron los estimadores no paramétricos Chao 1, estimador basado en abundancia, y Chao 2, estimador basado en incidencia. Para realizar estos análisis se utilizó el programa Primer 6 (Clarke \& Gorley, 2006).

\section{Resultados y discusión.}

Se registraron 16422 individuos pertenecientes a 39 especies (14 431 individuos de 35 especies en la temporada húmeda y 1991 individuos de 28 especies en temporada seca) (Tabla 1); 10 taxa fueron identificados al menos a nivel de género, esto debido a que los géneros Canthidium y Uroxys poseen muchas especies aún no descritas para el Neotrópico, además de ser taxonómicamente complicados de identificar a un nivel especifico. Onthophagus haematopus Harold 1875 fue la especie dominante, lo que concuerda con la literatura, donde la citan como una especie bien representada en los bosques amazónicos (Quintero \& 
Tabla 1. Lista y número de ejemplares colectados de escarabajos Scarabaeinae en lluvias y secas en Ucayali, Loreto. Se señala su gremio alimentario (G: generalista N: necrófago, C: coprófago, *: muestra preferencia); ND: no determinado. Datos de enero y octubre de 2013.

\begin{tabular}{|c|c|c|c|c|c|}
\hline \multirow[t]{2}{*}{ Especies } & \multicolumn{2}{|c|}{$\begin{array}{c}\text { Temporada de lluvias } \\
\text { (enero) }\end{array}$} & \multicolumn{2}{|c|}{$\begin{array}{l}\text { Temporada seca } \\
\text { (octubre) }\end{array}$} & \multirow[t]{2}{*}{ Preferencia alimenticia } \\
\hline & Copro & Necro & Copro & Necro & \\
\hline \multicolumn{6}{|c|}{ Tribu Ateuchini } \\
\hline Canthidium sp. 1 & 10 & 17 & 25 & & G \\
\hline Canthidium sp. 2 & 15 & 4 & 2 & 1 & G \\
\hline Canthidium sp. 3 & 382 & 142 & 1 & & G \\
\hline Canthidium sp. 4 & 233 & 66 & 1 & & $\mathrm{G}$ \\
\hline Canthidium sp. 5 & 39 & 10 & 2 & & G \\
\hline Canthidium kiesenwetteri (Harold, 1867) & 41 & 147 & & & G \\
\hline Canthidium onitoides (Perty, 1830) & 235 & 64 & 14 & & G \\
\hline Uroxys sp. 1 & 30 & 20 & 1 & & G \\
\hline Uroxys sp. 2 & 5 & 5 & 4 & & G \\
\hline Uroxys sp. 3 & 16 & 12 & 2 & & G \\
\hline Uroxys sp. 4 & 4 & & & & $\mathrm{C}(*)$ \\
\hline Uroxys sp. 5 & & & 1 & & $\mathrm{~N}(*)$ \\
\hline \multicolumn{6}{|c|}{ Tribu Canthonini } \\
\hline Canthon aequinoctialis (Harold 1868) & 2489 & 1002 & 181 & 3 & G \\
\hline Canthon brunneus (Schmidt, 1922) & 47 & 2 & & & G \\
\hline Canthon quinquemaculatus (Castelnau, 1840) & 251 & 799 & 19 & 205 & $\mathrm{G}$ \\
\hline Canthon juvencus (Harold, 1868) & & 2 & & & $C(*)$ \\
\hline Canthon mutabilis (Lucas, 1857) & 4 & & 1 & & $C(*)$ \\
\hline Canthon sericatus (Schmid, 1922) & 6 & 2 & & & G \\
\hline Coprophanaeus telamon (Erichson, 1847) & & & & 4 & $\mathrm{~N}(*)$ \\
\hline Deltochilum amazonicum (Bates, 1887) & 1 & & 1 & & $\mathrm{C}(*)$ \\
\hline Deltochilum nr. peruanum & 388 & 436 & 9 & 53 & G \\
\hline Pseudocanthon sp.1 & & & 1 & & $C(*)$ \\
\hline \multicolumn{6}{|c|}{ Tribu Coprini } \\
\hline Dichotomius mamillatus (Felsche,1901) & 240 & 144 & 16 & & G \\
\hline Dichotomius worontzowi (Pereira, 1942) & 2 & & & & $\mathrm{C}(*)$ \\
\hline Ontherus pubens (Génier, 1996) & 78 & 27 & 24 & & G \\
\hline \multicolumn{6}{|c|}{ Tribu Deltochini } \\
\hline Scybalocanthon nr. aereus & 20 & 44 & & & $\mathrm{G}$ \\
\hline Scybalocanthon imitans (Harold, 1868) & 75 & 169 & 2 & & $\mathrm{G}$ \\
\hline Scybalocanthon zischkai (Martinez, 1949) & 108 & 104 & 20 & 6 & G \\
\hline Scybalocanthon aff. moniliatus & & & 17 & 2 & G \\
\hline Sylvicanthon bridarollii (Martinez, 1949) & 3 & 1 & & & G \\
\hline \multicolumn{6}{|c|}{ Tribu Eurysternini } \\
\hline Eurysternus caribaeus (Herbst, 1789) & 4 & 3 & 2 & & G \\
\hline Eurysternus cayennensis (Castelnau, 1840) & 11 & 28 & & & G \\
\hline Eurysternus gracilis (Génier, 2009) & & 7 & & & $\mathrm{~N}(*)$ \\
\hline Eurysternus foedus (Guérin-Méneville, 1844) & 52 & 1 & 2 & & G \\
\hline Eurysternus hamaticollis (Balthasar, 1939) & 175 & 3 & 7 & & G \\
\hline Gromphas amazonica (Bates, 1870) & 5 & 2 & 123 & 2 & G \\
\hline Oxysternon lautum (Macleay, 1819) & 43 & 3 & 3 & & G \\
\hline \multicolumn{6}{|c|}{ Tribu Onthophagini } \\
\hline Onthophagus haematopus (Harold, 1875) & 5654 & 493 & 1233 & 1 & G \\
\hline Onthophagus marginicollis (Harold, 1880) & 4 & 2 & & & $\mathrm{G}$ \\
\hline Riqueza & 33 & 31 & 27 & 9 & ......... \\
\hline Abundancia & 10703 & 3792 & 1741 & 277 & ….............. \\
\hline
\end{tabular}

Halffter, 2009). Los Onthophagus americanos ocupan una gran variedad de bosques y usan una amplia variedad de recursos (Hanski \& Cambefort, 1991). También se registraron varias especies características de bosques inundables como Canthon quinquemaculatus Castelnau 1840, Ontherus pubens Génier 1996, Canthidium onitoides Perty 1830, especie necrófago común, y Canthon aequinoctialis
Harold 1868, especie generalista frecuente hasta 950 msnm (Larsen, 2004).

La riqueza $\left(F_{1,12}=4,2, p=0.06\right)$ no mostró diferencias significativas entre las dos temporadas y los dos tipos de cebo (Figura 3), posiblemente se debe a que los Scarabaeinae de los bosque neotropicales en su mayoría son generalistas (Halffter et al., 1992), se alimentan principalmente de fecas de mamíferos, siendo un tipo de alimento que fluctúa relativamente 
Tabla 2. Riqueza y abundancia por unidad de muestreo.

\begin{tabular}{lcc}
\hline \multicolumn{1}{c}{ Unidad de muestreo } & Riqueza & Abundancia \\
\hline Fila 1 de 10 coprotrampas & 26 & 3999 \\
Fila 2 de 10 coprotrampas & 27 & 2621 \\
Fila 3 de 10 coprotrampas & 29 & 2690 \\
Fila 4 de 10 coprotrampas & 23 & 1360 \\
Fila 5 de 10 necrotrampas & 22 & 1380 \\
Fila 6 de 10 necrotrampas & 19 & 583 \\
Fila 7 de 10 necrotrampas & 24 & 1040 \\
Fila 8 de 10 necrotrampas & 17 & 758 \\
& Temporada seca & \\
Fila 1 de 10 coprotrampas & 16 & 442 \\
Fila 2 de 10 coprotrampas & 14 & 250 \\
Fila 3 de 10 coprotrampas & 14 & 530 \\
Fila 4 de 10 coprotrampas & 20 & 492 \\
Fila 5 de 10 necrotrampas & 4 & 58 \\
Fila 6 de 10 necrotrampas & 8 & 124 \\
Fila 7 de 10 necrotrampas & 3 & 51 \\
Fila 8 de 10 necrotrampas & 4 & 44 \\
\hline
\end{tabular}

poco en cantidad comparado con frutos, semillas y detritos de árboles, flores y néctar (Janzen, 1983; Hanski, 1989).

La abundancia $\left(F_{1,12}=5,8, p=0.03\right)$ mostró diferencias significativas entre las dos temporadas y los dos tipos de cebo (Figura 3). Los Scarabaeinae presentan una estrecha relación con el suelo y es vital

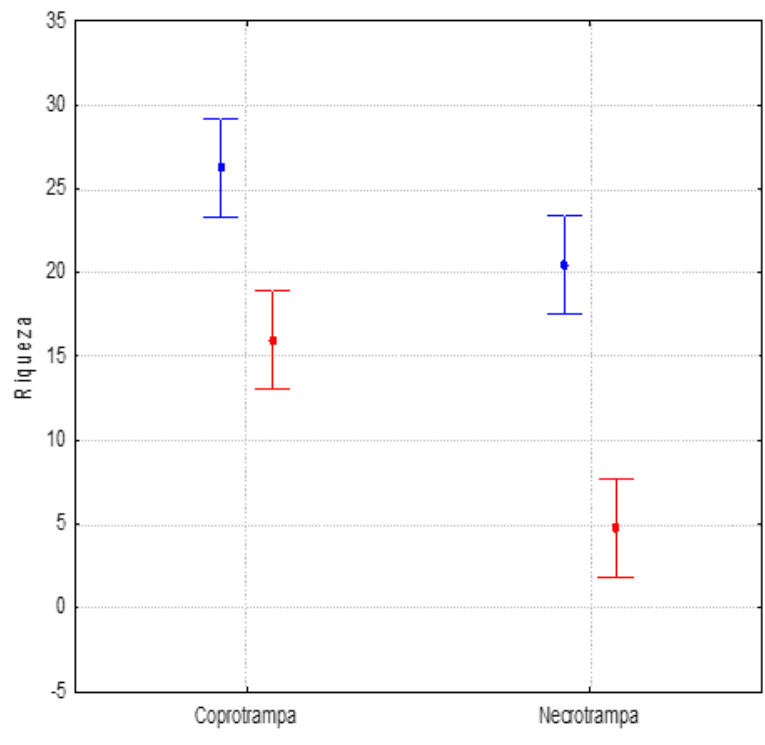

A.

Tipo de cebo

Figura 3. Variación temporal (media \pm EE) de la riqueza (A) y abundancia (B) de escarabajos Scarabaeinae en Sarayacu. En azul se representan los datos de la temporada de lluvias y en rojo, los de la seca. para su ciclo de vida, ya que es donde localizan su alimento, se desarrollan todos los estadíos inmaduros de cada especie (Halffter, 1991). Aunque es lógico pensar que exista una disminución de su abundancia como consecuencia de la destrucción de sus nidos y dificultad para obtener sus alimentos, sin embargo, se registró una marcada fluctuación de la abundancia debido a la temporalidad. Según Peck y Forsyth (1982) señalan que no observaron evidencias de temporalidad entre los Scarabaeinae en un bosque húmedo tropical, es decir, que el rango de clasificación de especies abundantes permaneció constante en la transición de las temporadas seca y húmeda en Rio Palenque al Oeste de Ecuador, explicándose que la temporada seca en Rio Palenque es ligeramente más seca que en la temporada húmeda, donde no existe un contrastante en la variación climática, a diferencia de las regiones templadas, lo que permite mayor especificidad y nicho estrecho a los insectos, reproducción continúa, y exclusiones adaptativas como una medida de coexistencia (Hanski \& Kuuseala, 1983). Sin embargo, los resultados del presente trabajo coinciden con Wolda (1978) quien señala que los insectos tropicales exhiben una gran variabilidad en su estacionalidad y año a año existen cambios en la abundancia aunque existen diferencias entre especies pero también dentro de especies dependientes de las condiciones locales. Howden \& Young (1981) señalan que muchas especies son más abundantes en una fase particular de la temporada húmeda. Estos Scarabaeinae están siendo afectados por las lluvias (Hanski \& Cambefort, 1991), con diferencias en abundancias y estructura de gremios entre las temporadas seca y húmeda (Andresen, 2005).

La composición de especies, según el análisis de

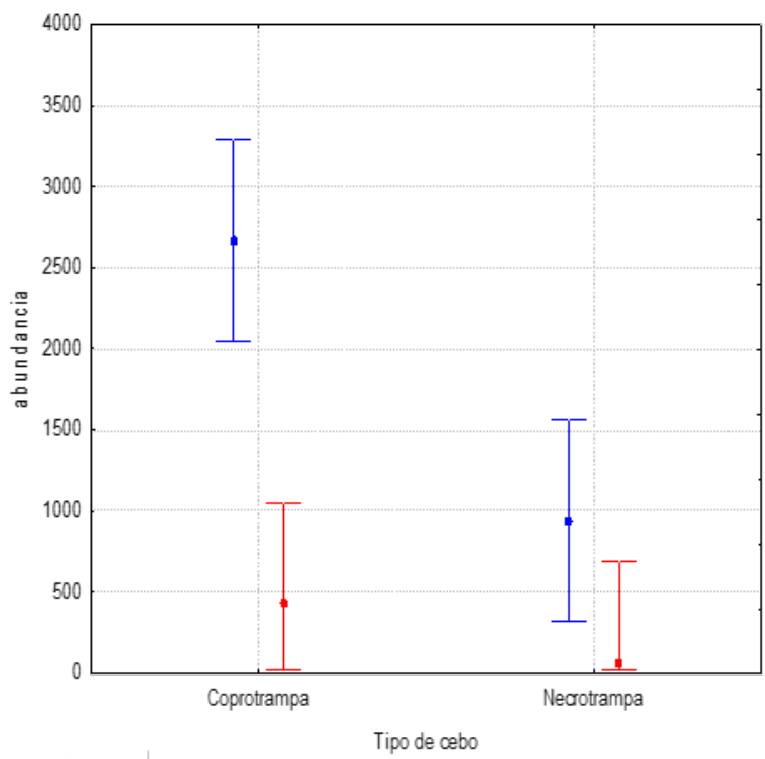

B. 
similitud (ANOSIM) es afectada significativamente por la temporada $(R=1, p=0.02)$ y por el tipo de cebo $(R=0.92, p=0.01)$. Concordando con Horgan (2006), quien sugiere que existen diferencias en patrones de agregación entre las temporadas lluviosa y seca y es difícil sugerir por qué ocurre esto, ya que los mecanismos que conducen a la agregación son aún desconocidos, pero posiblemente están relacionados a los cambios fisiológicos (incluyendo condiciones reproductivas) de los escarabajos entre periodos de muestreo, fluctuación de las densidades de parches naturales o cambio en la estructura del hábitat.

La mayoría de las especies registradas son generalistas (Tabla 1, Figura 3), lo cual este patrón es
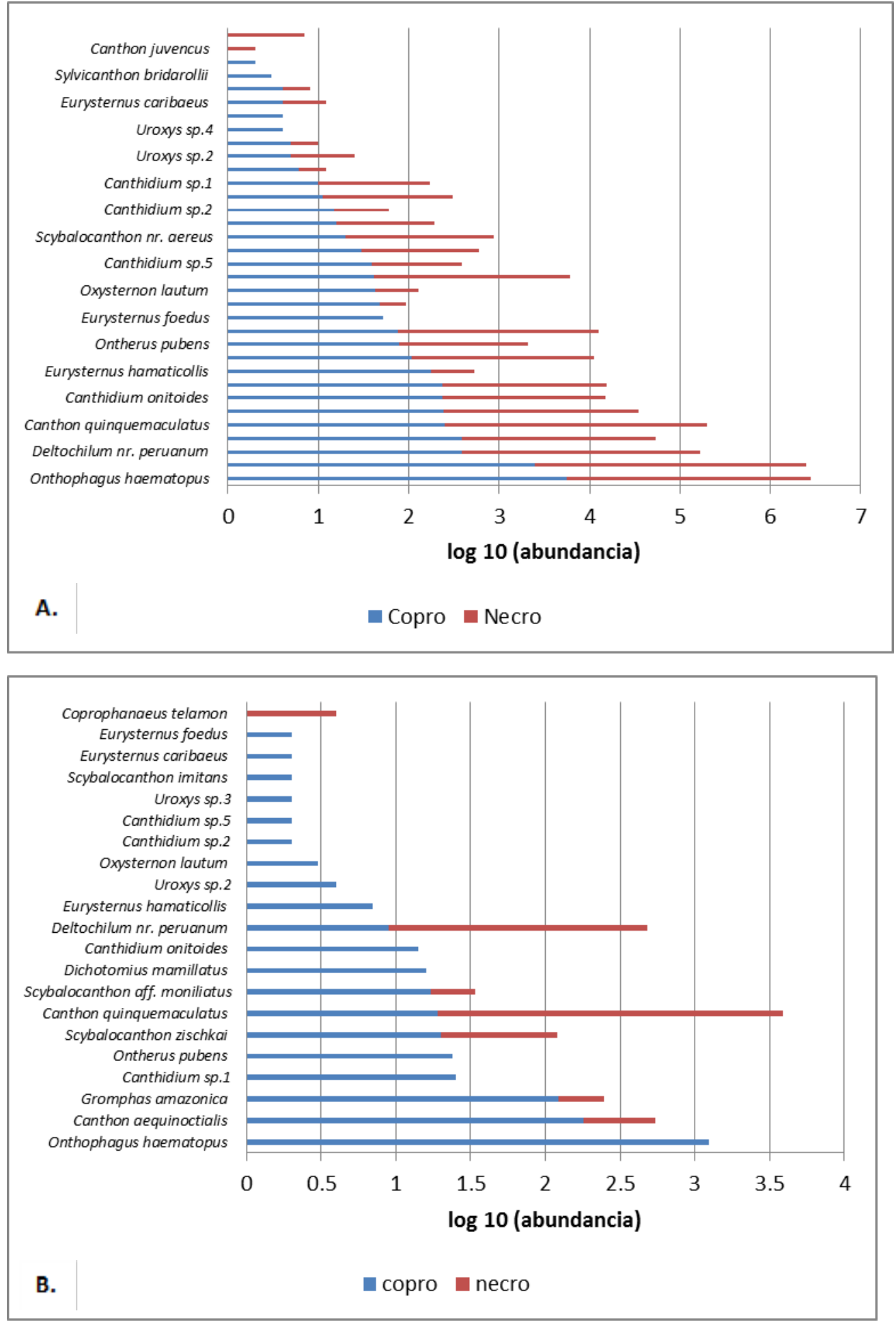

Figura 4. Abundancia $\left(\log _{10}\right)$ de cada especie de escarabajos Scaranaeinae colectados en coprotrampas (azul) y necrotrampas (rojo) en Sarayacu, Loreto. (A) temporada de lluvias, (B) temporada seca. 
Transform: $\log (X+1)$ Resemblance: S17 Bray Curtis similarity 3D Stress: 0.01

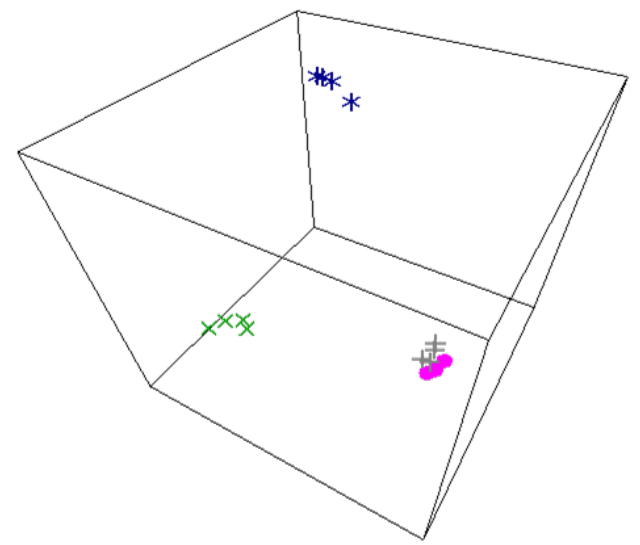

Figura 5. Análisis de escalamiento multidimensional (MDS) que muestra una similaridad de Bray Curtis de 60\% entre las comunidades de escarabajos Scarabaeinae en Sarayacu, Loreto. *azul: temporada seca en coprotrampas; $\mathbf{x}$ verde, temporada de lluvias con coprotrampas; + temporada de lluvias con necrotrampas; o rosado, temporada de lluvias con coprotrampas.

común en comunidades de Scarabaeinae de los bosques Neotropicales (Halffter et al., 1992). Además, se observó mayor atracción del cebo de fecas humanas, lo que concuerdan con Larsen et al. (2006), quienes refieren que las fecas de omnívoro constituyen el más eficiente atrayente para casi todas las especies de Scarabaeinae, y es atribuido a que tiene alto contenido de nitrógeno (Holter \& Scholtz, 2007). Asimismo, la competencia agresiva por el alimento en la temporada lluvias se atribuye a la inundación del bosque y se refleja en el análisis de agrupamiento de los escarabajos Scarabaeinae utilizando el método de escalamiento multidimensional (MDS) (Figura 5), donde no hubo diferenciación en la preferencia de tipo de cebo y se aprecia un hacinamiento, por lo contrario, en la temporada seca hubo dos grupos bien definidos donde se agruparon por los tipos de cebo, explicada por presentar menor competencia, y mayor desplazamiento en el bosque por no estar inundado. La alta densidad de la población y la escasez de los recursos (fecas de mamíferos y/o material orgánico en descomposición) generan una agresiva competencia e interfiere con el comportamiento de muchos Scarabaeinae (Peck \& Forsyth, 1982), lo que ocasionó una notable diferencia entre ambas temporadas. De manera similar, los mamíferos terrestres durante las lluvias se restringen a los espacios pequeños, o bien, tienden a moverse fuera de los hábitats inundables (Aquino et al., 2001). Aunque aún no está claro sobre las estrategias que tienen los escarabajos Scarabaeinae para afrontar la inundación de estos tipos de bosques, especulamos que el comportamiento de perchan sobre las hojas, les permite mantenerse a buen recaudo, así como localizar sus alimentos de manera rápida.

Finalmente, la curvas de acumulación de especies empleando los estimadores no paramétricos Chao 1 y Chao 2, sugieren que se tuvo un esfuerzo adecuado al tener una eficiencia de muestreo mayor a 94\% (Figura

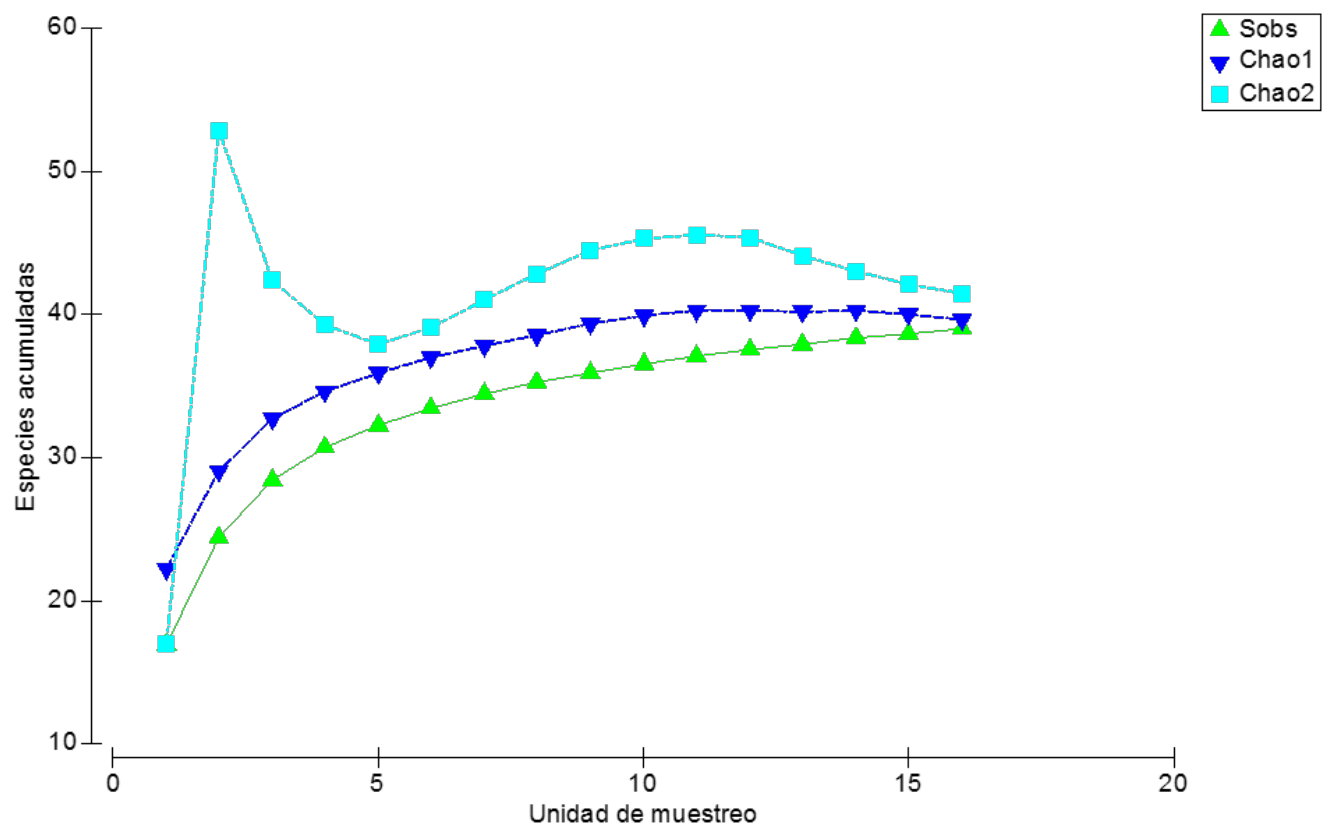

Figura 6. Curva de acumulación de especies con los indicadores no paramétrico Chao 1 (azul): Chao 2 (celeste); Especies observadas (verde) en Saravacu, Loreto. 
6, Tabla 3), coincidiendo con la literatura, donde los señalan como un buen grupo para ser estudiado, por su método sencillo de colecta, además de obtener en corto tiempo muestras representativas de riqueza presentes en una localidad (Villarreal et al., 2006).

Tabla 3. Eficiencia de muestreo (en porcentaje) empleando los estimadores no paramétricos Chao 1 y Chao 2.

\begin{tabular}{cc}
\hline Estimador & Total \\
\hline Chao 1 & $98.2 \%$ \\
Chao 2 & $94 \%$ \\
\hline
\end{tabular}

\section{Conclusiones.}

Con base en los resultados y discusión, se concluye que:

1. Se registró en total 39 especies de Scarabaeinae en un bosque de llanura inundable de Loreto, Perú, de las cuales, Ontophagus haematopus, Canthon quinquemaculatus y $C$. aequinoctialis fueron las especies dominantes, además se registró 29 de especies generalistas.

2. El cebo de fecas humanas fue más efectivo para la atracción de escarabajos Scarabaeinae, este poder de atracción y es atribuido a que tiene alto contenido de nitrógeno.

3. La abundancia y composición de escarabajos Scarabaeinae mostraron diferencias significativas entre ambas temporadas y tipo de cebo. Por lo contrario, la riqueza no fue significativo.

4. Se comprobó que existe una marcada fluctuación en la abundancia de escarabajos Scarabaeinae debido a la temporalidad.

\section{Agradecimientos.}

Un agradecimiento a la Fundación Ideawild por financiar parte de la salida de campo y materiales de laboratorio. Al Ministerio de Agricultura que brindó el Permiso de Colecta RD No 0237-2013- MINAGRIDGFFS/DGEFFS. Al Servicio Nacional de Meteorología e Hidrología (Senamhi) por brindarnos datos climatológicos de la Estación Contamana. A la Dra. Diana Silva por sus comentarios, al Mg. Jorge Peralta por su asesoramiento estadístico y a la familia Dávila por permitirnos realizar colectas en su terreno.

\section{Literatura citada.}

Adis J. 1986. An "aquatic" millipede from a Central Amazonian inundation forest. Oecologia, 68: 347-349.

Adis J. 1988. Comparative ecological studies of the terrestrial arthropod fauna in central Amazonian inundation forests. Amazonia, 7: 87-173.

Aquino R., Bodmer R. \& Gil J. 2001. Mamíferos de la Cuenca del río Samira: Ecología poblacional y sustentabilidad de la caza. Programa Integral de Desarrollo y Conservación Pacaya Samiria, Lima, Perú. 140 pp.
Andresen E. 2005. Effects of season and vegetation type on community organization of dung beetles in a tropical dry forest. Biotropica, 37: 291-300.

Arellano L., León-Cortés J. \& Halffter G. 2008. Response of dung beetle assemblage to landscape structure in remnant natural and modified habitats in southern Mexico. Insect Conservation and Diversity 1:253-262.

Clarke K.R. \& Warwick R.N. 2001. Change in marine communities: an approach to statistical analysis and interpretation, 2a. edición. PRIMER-E, Plymouth. 172 pp.

Clarke K.R \& Gorley R.N. 2006. PRIMER (Plymouth Routines in Multivariate Ecological Research) 5.2.2. User Manual/Tutorial.

Estrada A. \& Coates-Estrada R. 1991. Howler monkey (Alouatta palliata), dung beetles (Scarabaeidae) and seed dispersal: Ecological interactions in the tropical rain forest of Los Tuxtlas, Veracruz, México. Journal of Tropical Ecology, 7(3): 459-474.

Halffter G. 1991. Historical and ecological factors determining the geographical distribution of beetles (Coleoptera: Scarabaeidae: Scarabaeinae). Folia Entomológica Mexicana, 82: 195-238.

Halffter G., Favila M.E. \& Halffter V. 1992. A comparative study on the structure of scarab guilds in tropical rainforests and derived ecosystems. Folia Entomológica Mexicana, 84: 131-156.

Hanski I. 1989. Dung beetles. En H. Lieth \& M. J. Werger (eds.). Tropical rain forest ecosystems. Elsevier Science, Paises Bajos, Holanda, 489-511.

Hanski I. \& Cambefort Y. 1991. Dung beetle ecology. Princeton University Press, Nueva Jersey, Estados Unidos, 481 pp.

Hanski I. \& Kuusela S .1983. Dung beetle communities in the Åland archipelago. Acta Entomologica Fennica, 42:36-42.

Holter P. \& Scholtz.2007 C. What do dung beetles eat? Ecological Entomology, 32:690-697.

Horgan, F. 2006. Aggregation and coexistence of dung beetles in montane rain forest and deforested sites in central Peru. Journal of Tropical Ecology, 22: 359-370.

Howden H.F \&. Young O.P. 1981. Paramanian Scarabaeinae: taxonomy, distribution and habitats (Coleoptera: Scarabaeidae). Contribution American Entomology Institute, 18: 204 pp.

Instituto de Investigación de la Amazonia Peruana IIAP. 2004. Diversidad de Vegetación de la Amazonia Peruana expresada en un mosaico de imágenes de satélite-BIODAMAZ. Documento técnico No 12. Iquitos, Perú.

Instituto Nacional de Recursos Naturales INRN. 1996. Guía explicativa del mapa forestal 1995. INR-49-DGF. Lima, Perú. 147 pp.

Janzen D. H. 1993. Caterpillar seasonality in a Costa Rican dry forest. Páginas 448-477. En: Caterpillars. Ecological and evolutionary constraints on foraging, N. E. Stamp and T. M. Casey (eds.) Chapman and Hall, Nueva York, EEUU.

Larsen T.H. 2004. Dung Beetles /Escarabajos peloteros. Páginas 185-192. En: C. Vriesendorp, L. Rivera Chavez, D. Moskovits y J. Shopland (editores.) Peru: Megantoni. rapid biological inventories. Report 15: The Field Museum, Chicago, Illinois, EEUU. 
Larsen T.H., Lopera A. \& Forsyth, A. 2006. Extreme trophic and habitat specialization by Peruvian dung beetles. Coleopterist's Bulletin, 60(4): 315-324.

Miller A. 1954. Dung beetles (Coleoptera, Scarabaeidae) and of insects in relation to human feces in a hookworm area of South Georgia. America Journal Tropical Medical, 3(2): 372-388.

Oficina Nacional de Evaluación ONE. 1976. Mapa ecológico del Perú-Guía explicativa. Lima.

Peck S.B. \& Forsyth. A. 1982. Composition, structure, and competitive behavior in a guild of Ecuadorian rain-forest dung beetles (Coleoptera, Scarabaeidae). Canadian Journal of Zoology-Revue Canadienne de Zoologie, 60: $1624-1634$.

Quintero I. \& Halffter G. 2009. Temporal changes in a community of dung beetles (Insecta: Coleoptera: Scarabaeinae) resulting from the modification and fragmentation of tropical rain forest. Acta Zoológica Mexicana (n.s.), 25: 625-649.

Quintero I. \& T. Roslin. 2005. Rapid recovery of dung beetles communities following habitat fragmentation in Central Amazonia. Ecology, 86(12): 3303-3311.

Servicio Nacional de Meteorología e Hidrología del Perú SENAMHI. 2013. Datos meteorológicos de la Estación Contamana. Lima, Perú.
Silva P.G. 2011. Dung beetles (Coleoptera: Scarabaeidae: Scarabaeinae) of two non- native habitats in Bagé, Rio Grande do Sul, Brazil. Zoological Studies, 50(5): 546559.

Steinfeld H.P, Gerber T., Wassenaar V., Castel M. Rosales \& De Haan C. 2006. Livestock's long shadow: environmental issues and options. Food and Agriculture Organization of the United Nations, Roma, Italia, 414 pp.

StatSoft Inc. 2007. STATISTICA (data analysis software system), version 8.1. StatSoft, Inc.,Tulsa, OK, EE.UU.

Vaz-de-Mello F.Z., Edmonds .W.D., Ocampo F. \&. Schoolmeesters P. 2011. A multilingual key to the genera and subgenera of the subfamily Scarabaeinae of the New World. Zootaxa, 2854: 1-73.

Villareal H., Álvarez M., Córdoa S., Escobar F., Fagua G., Gast F., Mendoza M., Ospina H. \& Umaña A.M. 2006. Manual de métodos para el desarrollo de inventarios de biodiversidad. Programa de inventarios de biodiversidad. Instituto de Investigación de Recursos Biológicos Alexander von Humbolt. Bogotá, Colombia. 230 pp.

Wolda H. 1978. Seasonal fluctuations in rainfall, food and abundance of tropical insects. Journal of Animal Ecology, 47: 369-381.

\footnotetext{
${ }^{1}$ Departamento de Entomología, Museo de Historia Natural. Universidad Nacional Mayor de San Marcos. Av. Arenales 1256. Apartado 14-0434, Lima-14, Perú. E-mail: lidia.sulca@gmail.com.

${ }^{2}$ Laboratorio de Invertebrados Acuáticos Continentales. Universidad Nacional Mayor de San Marcos. E-mail: ahuamantinco@hotmail.com.
} 